\title{
Routing Protocol in Wireless Sensor Network: A Review
}

\author{
Swati Jaryal \\ M.Tech (Scholar) \\ CGC, Landran
}

\author{
Devendra Prasad, PhD \\ Professor \\ CGC, Landran
}

\author{
Amit Verma, $\mathrm{PhD}$ \\ Professor \\ CGC, Landran
}

\begin{abstract}
Current advances in wireless sensor networks (WSNs) have led to various routing protocols specifically designed for sensor networks where power consumption is an important concern. Sensor Nodes (SNs) in WSNs limited in terms of computing power, transmission range and energy as they are battery operated. When these SNs are deployed in hostile environment where reach-ability is not possible they can not be recharge so, protocols in WSNs should be developed keeping in view the energy consumption. Routing protocols for wireless sensor network are responsible for maintaining the routes in the network and have ensured reliable multi- hop communication. This paper discussed some energy-efficient routing protocols in WSNs.
\end{abstract}

\section{Keywords}

Wireless Sensor Networks, Sensor Nodes, Energy efficiency, Cluster Head.

\section{INTRODUCTION}

A wireless sensor network (WSNs) is widely considered as one of the most important technologies for the twenty-first century [1]. In the past decades, it has received tremendous attention from both academia and industry all over the world. A WSN typically consists of a large number of low-cost, lowpower, and multifunctional wireless Sensor Nodes (SNs), with sensing, wireless communications and computation capabilities [2, 3]. These SNs communicate over short distance via a wireless medium and collaborate to accomplish a common task, for example, environment monitoring, military surveillance, and industrial process control [4].

The basic philosophy behind WSNs is that, while the capability of each individual SNs is limited, the aggregate power of the entire network is sufficient for the required mission. In many WSNs applications, the deployment of SNs is performed in an ad hoc fashion without careful planning and engineering. Once deployed, the SNs must be able to autonomously organize themselves into a wireless communication network. SNs are battery-powered and are expected to operate without attendance for a relatively long period of time. In most cases it is very difficult and even impossible to change or recharge batteries for the sensor nodes. WSNs are characterized with denser levels of SNs deployment, higher unreliability of SNs, and sever power, computation, and memory constraints.

Thus, the unique characteristics and constraints present many new challenges for the development and application of WSNs. A large number of research activities have been carried out to explore and overcome the constraints of WSNs and solve design and application issues.

In this paper various routing protocols for WSNs are discussed and compared. Finally, concludes the review.

\section{RELATED WORK}

In [5] authors solve the problem of resource wastage in routing when two real time data flows on the same route. An author sets a time variable called $\mathrm{P}$ time to determine the life time of port. When a route is overtime, it is considered available again and should release routing resource timely. Then routing resources are allocated reasonably and can meet the demand of WSN Quality of Service (QoS). Routing optimization can effectively reduce node cost and prolong node life time in WSNs.

In [3] authors defined a scheduled based routing protocol that uses both topological and link quality information to adapt to environment changes. The multiple channels are used to enable parallel transmissions and hence allow fast data gathering at the sink while reducing collisions. Its aim is to optimize the choice of the next-hop node in order to have a better overall efficiency and a better load balancing in the network.

In [6] authors defined that Sensor network nodes are limited with respect to energy supply, restricted computational capacity and communication bandwidth. Most of the attention, however, has been given to the routing protocols since they might differ depending on the application and network architecture. To prolong the lifetime of the sensor nodes, designing efficient routing protocols is critical. Even though sensor networks are primarily designed for monitoring and reporting events, since they are application dependent, a single routing protocol cannot be efficient for sensor networks across all applications.

They analyse the design issues of sensor networks and present a classification and comparison of routing protocols. This comparison reveals the important features that need to be taken into consideration while designing and evaluating new routing protocols for sensor networks.

In [7] authors principally focus in one attack that is HELLO flood attack. Some directing conventions require to visible hello message intermittently to its neighbour nodes. These SNs gets this welcome message accept that the sender is in its range.

Table 1: Various Technique Used and Conclusion of Existing Work

\begin{tabular}{|l|l|l|}
\hline $\begin{array}{c}\text { Sr. } \\
\text { No. }\end{array}$ & \multicolumn{1}{|c|}{$\begin{array}{c}\text { Techniques } \\
\text { Used }\end{array}$} & \multicolumn{1}{c|}{ Conclusion } \\
\hline 1 & $\begin{array}{l}\text { WSN in Quality } \\
\text { of Services }\end{array}$ & $\begin{array}{l}\text { can successfully reduce routing } \\
\text { waste }\end{array}$ \\
\hline 2 & $\begin{array}{l}\text { Hybrid Genetic } \\
\text { Algorithm }\end{array}$ & $\begin{array}{l}\text { balances energy consumption and } \\
\text { covers the network life cycle; the } \\
\text { network efficiency of WSN is } \\
\text { improved }\end{array}$ \\
\hline
\end{tabular}




\begin{tabular}{|l|l|l|}
\hline 3 & $\begin{array}{l}\text { Minimum Hop } \\
\text { Routing }\end{array}$ & $\begin{array}{l}\text { To balance the energy consumption } \\
\text { of the nodes. }\end{array}$ \\
\hline 4 & $\begin{array}{l}\text { Flood Attack, } \\
\text { and } \\
\text { Bidirectional } \\
\text { Verification } \\
\text { Scheme. }\end{array}$ & $\begin{array}{l}\text { Routing scheme RAEED provide } \\
\text { better presentation in the presence } \\
\text { of DoS attacker. The number of } \\
\text { messages } \\
\text { Swapped among the nodes and time } \\
\text { to comprehensive key setup phase is } \\
\text { low for RAEED. }\end{array}$ \\
\hline
\end{tabular}

Authors presented a LEACH protocol in [8]. The LEACH is one of the prominent and well recognized protocols in WSNs. The main purpose of this protocol is to increase the lifespan of WSNs by lowering their energy. LEACH is a division protocol which works in binary phase's set-up phase and steady-state phase.

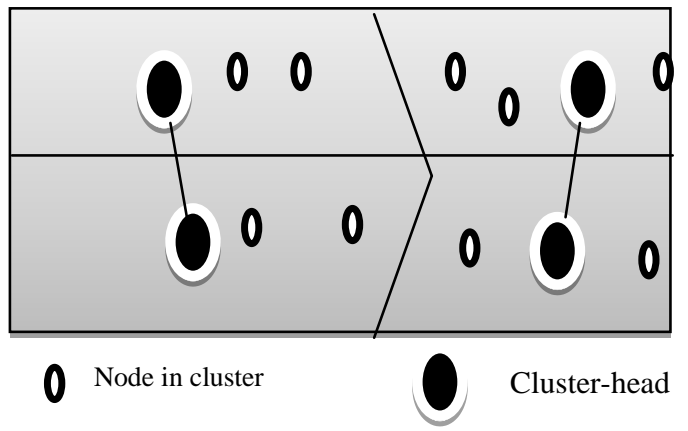

Base Station

Figure 1: LEACH Protocol Structure.

- In First phase, all SNs in the deployment area form cluster regions. Then they select the Cluster Head $(\mathrm{CH})$ for each cluster with the maximum energy of SNs.

- In second phase the $\mathrm{CH}$ acts as a region and all members of the cluster region sends the collected data to the $\mathrm{CH}$ in their allotted time-division multiple access slots.

Advantages of LEACH protocol:

- The $\mathrm{CH}$ combines the complete data which lead to optimize the traffic in the entire-network.

- There is an individual hop-routing from nodes to $\mathrm{CH}$ for saving energy.

- It increases the life-time of the sensor network.

Disadvantages of LEACH protocol:

- LEACH doesn't give any idea about the number of CHs in the network.

- The highest demerit of LEACH is that when due to any reason $\mathrm{CH}$ dies, the cluster would become useless because the data gathered by the cluster-nodes will never reach its sink i.e. BS (Base Station) [9]

The objective of PEGASIS (Power Efficient Gathering in Sensor Information System) for individual node is to receive from and transit to the nearest neighbours and take-turns for transmission to the base station (Main Head). This approach will divide the energy load among the SNs in the particular network [10]. After that the SNs are randomly placed in the plane area and therefore, the $\mathrm{I}^{\text {th }}$ node is at a random position.

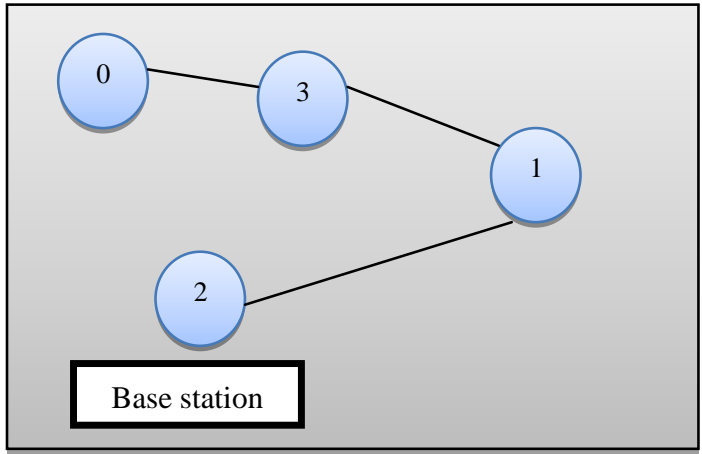

Figure 2: Chain Construction in PEGASIS protocol.

The SNs would be organised to form a chain, which could either be accomplished by the node themselves using a greedy- algorithm starting from any node. Alternatively, the BS could calculate this chain and broadcast it to every SNs. For assembling information in each round, each node receives data from individual neighbour, fused with its own data and transmits to the other nearest node in the chain.

The below defined protocols are various versions of PEGASIS [13]:

- Energy Efficient PEGASIS Based (EEPB): It is an enhanced PEGASIS algorithm in WSNs. In PEGASIS greedy algorithm is used to form the data chain, it can result in communication distance between two sensors presents too long. Thus, the sensors consume more energy in transmitting the data and die early. In the chaining process, a node will consider the average distance of which the chain is designed. This distance is known as separate distance. If the distance from the closest node to the upstream node is longer than thresh distance, the closest node is the "far node". If the closest node joins the chain, it will be "long chain". EBPEGASIS avoids this phenomenon using distance threshold. It not only saves energy on threshold, but also balances the energy consumption of all sensor nodes.

- H-PEGASIS: It is an extended version of PEGASIS protocol. It was introduced with the objective of decreasing the delay of transmission packets to the BS. It recommends a solution to data gathering problems by seeing energy $X$ delay metrics. In order to reduce delay, simultaneous data messages are transmitted. To avoid collisions, signal coding is designed e.g. CDMA to avoid signal distortion, only spatially detached nodes are acceptable transmit data at the same time. With CDMA capable nodes, the chain forms the tree structure like hierarchy and each certain node transmit the data to the node of upper hierarchy. This confirms parallel data broadcast and decreases the delay expressively [12].

Table 2: Existing Parameters Used

\begin{tabular}{|l|l|l|}
\hline $\begin{array}{c}\text { Sr. } \\
\text { no. }\end{array}$ & \multicolumn{1}{|c|}{$\begin{array}{c}\text { Approach } \\
\text { Name }\end{array}$} & \multicolumn{1}{c|}{ Parameters } \\
\hline 1. & $\begin{array}{l}\text { PEGASIS } \\
\text { Protocol }\end{array}$ & Number of Rounds \\
\hline 2. & $\begin{array}{l}\text { LEACH } \\
\text { Protocol }\end{array}$ & $\begin{array}{l}\text { Alive Nodes, Dead Nodes, } \\
\text { Data Gathered and energy } \\
\text { Split }\end{array}$ \\
\hline
\end{tabular}


PEGASIS with Double Cluster Head (PDCH) balances the load of every node and increase network lifetime. Generally, PEGASIS protocol uses one $\mathrm{CH}$ that communicates with the BS. Here instead of one, double $\mathrm{CHs}$ are used in a single chain and it gives a hierarchical structure so that long chaining is avoided. PDCH outperforms PEGASIS by eliminating dynamic cluster formation and reducing the distance between nodes. It also reduces the number of messages sent to and from other nodes and using only one transmission to BS per round. As the load is distributed among the nodes, the network lifetime increases and so improves the quality of network [13].

Improved Energy Efficient Pegasis Based (IEEPB) protocol this protocol, overcomes the deficiencies of EEPB. When EEPB builds a chain, the threshold adopted is uncertain and complex to determine. This results in the formation of "long chain". Also, when EEPB selects the leader, it ignores the node energy and the distance between the BS and node that optimizes the selection of leader. Based on this, IEEPB compares the distance between two nodes twice and finds the shortest path to link two adjacent nodes. The chain construction is simplified such that formation of "long chain" is avoided. Also, while selecting the leader, IEEPB considers the node's energy, distance between the BS and the node. Also normalizes these two factors and assigns different weight co-efficient to them. Finally, the node with the minimum weight becomes the leader. IEEPB has higher energy efficiency and hence longer network lifetime [13].

\section{CONCLUSION}

One of the main challenges in the design of routing protocols for WSNs is energy efficiency due to the scarce energy resources of sensors. The ultimate objective behind the routing protocol design is to keep the sensors operating for as long as possible, thus extending the network lifetime. The energy consumption of the sensors is dominated by data transmission and reception. Therefore, routing protocols designed for WSNs should be as energy efficient as possible to prolong the lifetime of individual sensors, and hence the network lifetime. In this paper, we have discussed the performance of various routing Protocols in WSNs. These protocols are developed keeping in view the energy-efficiency and performance. The process of data collection and fusion among clusters is also explored. It improves the dataaggregation efficiency and optimizes the energy consumption.

\section{REFERENCES}

[1] Kaschel, Hector, and Johanna Ortega. "Energy efficiency in routing protocols applied to WSN." In Automatica (ICA-ACCA), pp. 1-8. IEEE. 2016.

[2] Xibei, Jia, Zhang Huazhong, and Zhang Jingchen. "Research of data aggregation routing protocol in WSN data-related applications." In Computer Science and Information Technology (ICCSIT), in proceeding of 3rd IEEE International Conference on, vol. 1, pp. 647-651. 2010.
[3] Misson, Michel. "Multi-channel routing protocol for dynamic WSN." In Wireless Days (WD), march 23-25, pp. 1-3. 2016.

[4] Chen, Ming, and Ting-lei Huang. "A new algorithm of layered routing protocol in WSN." In Intelligent Computing and Integrated Systems (ICISS), pp. 942-943. 2010.

[5] Cui, Xiao-yan, and Zhao Liu. "An improved algorithm for flow-based QoS routing protocol in WSN." In International symposium on Microwave, Antenna, Propagation and EMC Technologies for Wireless Communications, Proceeding in 3rd IEEE International Symposium, pp. 745-747. 2009.

[6] Biradar, Rajashree V., V. C. Patil, S. R. Sawant, and R. R. Mudholkar. "Classification and comparison of routing protocols in wireless sensor networks." In Ubiquitous Computing Security Systems, vol. 4, Issue 2, pp. 704 711. 2009

[7] Maheswari, S. Uma, N. S. Usha, EA Mary Anita, and K. Ramaya Devi. "A novel robust routing protocol RAEED to avoid DoS attacks in WSN." In Information Communication and Embedded Systems (ICICES), pp. 1-5. 2016.

[8] Xiangning, Fan, and Song Yulin. "Improvement on LEACH protocol of wireless sensor network." Proceeding in SENSORCOMM international conference on sensor Technologies and Applications, vol. 00, pp. 260-264. 2007.

[9] Malik, Meena, Dr Yudhvir Singh, and Anshu Arora. "Analysis of LEACH protocol in wireless sensor networks." International Journal of Advanced Research in Computer Science and Software Engineering (IJARCSSE), vol. 3, Issue 2, pp 178-183. 2013.

[10] Lindsey, Stephanie, and Cauligi S. Raghavendra. "PEGASIS: Power-efficient gathering in sensor information systems." proceeding in IEEE Aerospace conference, vol. 3, pp. 1125-1130. 2002.

[11] Alpesh R.Sankalia "PEGASIS: Power Efficient Gathering in Sensor Information Systems."International journal of Electronics and Communication Engineering (IJSRST) Vol. 1, Issues 5, pp.108-112. 2015.

[12] Rana, Hetal, Sangeeta Vhatkar, and Mohommad Atique. "Comparative Study of PEGASIS Protocols in Wireless Sensor Network." IOSR Journal of Computer Engineering (IOSR-JCE) Vol. 16, Issue 5, pp. 25-30. 2014.

[13] Fengsen, Qi Bing, Tang Lianguri, “An Improved EnergyEfficient PEGASIS-Based Protocol in Wireless Sensor Networks", IEEE Eighth International Conference on Fuzzy System and Knowledge Discovery, pp. 22302233. 2011 